\title{
One-year symptom trajectories in patients with stable schizophrenia maintained on antipsychotics versus placebo: meta-analysis ${ }^{\dagger}$
}

\author{
Hiroyoshi Takeuchi, Navot Kantor, Marcos Sanches, Gagan Fervaha, Ofer Agid and Gary Remington
}

\section{Background}

As definitions of relapse differ substantially between studies, in investigations involving data aggregation, total scores on clinical rating scales provide a more generalisable outcome

\section{Aims}

To compare total symptom trajectories for antipsychotic versus placebo treatment over a 1-year period of maintenance treatment in schizophrenia.

\section{Method}

Randomised controlled trials with antipsychotic and placebo treatment arms in patients with stable schizophrenia that reported Positive and Negative Syndrome Scale and Brief Psychiatric Rating Scale total scores at more than one time point were included. Meta-regression analyses were employed using a mixed model.

\section{Results}

A total of 11 studies involving 2826 patients were included Meta-regression analyses revealed significant interactions between group and time (PS $<0.0001)$; both standardised total scores and per cent score changes remained almost unchanged in patients continuing antipsychotic treatment, whereas symptoms continuously worsened over time in those switching to placebo treatment.

\section{Conclusions}

When considering long-term antipsychotic treatment of schizophrenia, clinicians must balance symptomatic and functional outcomes.

\section{Declaration of interest}

H.T. has received manuscript fees from Sumitomo Dainippon Pharma. O.A. has received speaker's honoraria from Eli Lilly \& Company USA, Eli Lilly Canada, Janssen-Ortho (Johnson \& Johnson), Lundbeck, Mylan Pharmaceuticals, Novartis, Sepracor Inc. and Sunovion, and consultant fees from BMS, Eli Lilly \& Company USA, Eli Lilly Canada, Janssen-Ortho (Johnson \& Johnson), Lundbeck, Novartis, Otsuka, Roche, Sepracor Inc. and Sunovion, and research support from Boehringer Ingelheim, Neurocrine Biosciences, Janssen-Ortho (Johnson \& Johnson), Otsuka, Pfizer Inc. and Sunovion. G.R. has received research support from Novartis, Medicure and Neurocrine Bioscience, consultant fees from Laboratorios Farmacéuticos ROVI, Synchroneuron and Novartis, and speaker's fees from Novartis.

\section{Copyright and usage}

(c) The Royal College of Psychiatrists 2017
Antipsychotic treatment plays a critical role in alleviating the acute symptoms of schizophrenia and maintenance treatment is routinely recommended to prevent re-emergence/exacerbation of symptoms (i.e. relapse). ${ }^{1}$ For example, a recent meta-analysis conducted by Leucht et al, including 64 randomised controlled trials (RCTs), provides compelling evidence that antipsychotic maintenance treatment is superior to placebo in reducing the risk of relapse in stable schizophrenia. ${ }^{2}$ That said, definitions of relapse differ substantially between investigations, ${ }^{3}$ which in turn compromises validity when directly comparing multiple studies. This has been noted in Leucht et al's meta-analysis, ${ }^{2}$ in addition to others comparing efficacy for relapse prevention between first(FGAs) and second-generation antipsychotics (SGAs) ${ }^{4}$ as well as oral and long-acting injectable (LAI) antipsychotics. ${ }^{5}$ In the context of data aggregation, total scores (i.e. a continuous variable) on clinical rating scales such as the Positive and Negative Syndrome Scale (PANSS) ${ }^{6}$ and Brief Psychiatric Rating Scale $(\mathrm{BPRS})^{7}$ provide a more generalisable outcome than relapse, a dichotomous variable that can vary one definition to the next There have been several meta-analyses ${ }^{8-11}$ using PANSS or BPRS total scores, although all have investigated the onset of antipsychotic action or time course of symptom change during antipsychotic treatment in the acute phase of schizophrenia. To date, none has compared symptom trajectories between antipsychotic and placebo treatments in the maintenance phase.

${ }^{\dagger}$ See editorial, pp. 127-129, this issue.
Accordingly, the following question relating to maintenance treatment of schizophrenia remains unanswered: is antipsychotic treatment superior to a placebo replacement in terms of symptom trajectories in the maintenance phase of schizophrenia? To address this clinical question, we conducted a systematic review and meta-analysis of RCTs comparing symptom trajectories between antipsychotic and placebo treatments in stable patients with schizophrenia.

\section{Method}

\section{Literature search and study selection}

Our clinical question built upon the meta-analysis conducted by Leucht et al. ${ }^{2}$ Their meta-analysis included 64 RCTs with treatment arms consisting of an antipsychotic(s) along with placebo or no treatment, in patients with schizophrenia spectrum disorders (i.e. schizophrenia, schizoaffective disorder, and/or schizophreniform disorder) who had been stabilised on antipsychotics. From these 64 studies, we selected those that met the following additional eligibility criteria: (a) studies using the PANSS or BPRS; (b) studies reporting the PANSS or BPRS total scores at both baseline and another time point, at minimum; and (c) studies using a last-observation-carried-forward (LOCF) method.

Considering that Leucht et al performed their literature search several years ago, we conducted a systematic literature search (last search: 19 January 2016), using the MEDLINE, Embase, and Cochrane Central Register of Controlled Trials (CENTRAL) with 
a limitation of year (i.e. from 2011 to current) in order to update the search. We adopted the same search strategy used by Leucht et $a l^{2}$ including keywords ((cessation* OR withdraw* ${ }^{*}$ OR discontinu* OR halt ${ }^{\star}$ OR stop ${ }^{\star}$ OR drop-out ${ }^{\star}$ OR dropout ${ }^{\star}$ OR drop out OR rehospitalis* OR relaps* OR maintain* OR maintenance OR recur $^{\star}$ ) AND (schizophr ${ }^{\star}$ OR schizoaff $\left.{ }^{\star}\right)$ ) and a limitation ('randomized controlled trial'). Two authors (H.T. and N.K.) independently conducted the literature search in accordance with the PRISMA (Preferred Reporting Items for Systematic Reviews and Meta-analyses) statement. ${ }^{12}$ We selected studies that met the following eligibility criteria: (a) original studies (i.e. not protocols, reviews, meta-analyses or secondary analyses); (b) RCTs; (c) studies including treatment arms of both an antipsychotic(s) and a placebo or no treatment; (d) studies where $\geqslant 70 \%$ of participants were diagnosed with schizophrenia spectrum disorders; (e) studies including only patients with stable psychopathology; (f) studies using the PANSS or BPRS; (g) studies reporting the PANSS or BPRS total scores at both baseline and another time point, at minimum; and (h) studies using an LOCF method. Risk of bias for each included study was assessed according to the Cochrane Handbook for Systematic Reviews of Interventions. ${ }^{13}$

\section{Data extraction}

The PANSS or BPRS total scores were collected for each study for up to 52 weeks from randomisation; weekly for the first 4 weeks and at 4 -week intervals thereafter. In order to utilise as much data as possible, values not lining up with the 4-week intervals were moved forward to the next 4 -week value (i.e. a value at $4 n$ weeks +2 weeks was recorded as a value at $4(n+1)$ weeks). Data presented in graph-form were extracted automatically using an online computer program (Dexter; German Astrophysical Virtual Observatory, University of Heidelberg, Germany; available at http://dc.zah.uni-heidelberg.de/dexter/ui/ui/custom); for the one case in which the software could not retrieve the data, it was extracted manually instead.

\section{Data analysis}

Standardised total scores were calculated by dividing the PANSS or BPRS total scores by the number of items (i.e. 30 items for PANSS and 18 items for BPRS) and used as a primary outcome. Per cent score changes were also calculated using the following formulation ${ }^{14-16}$ as a secondary outcome: (PANSS or BPRS total score at a given time-PANSS or BPRS total score at baseline)/ (PANSS or BPRS total score at baseline -30 for PANSS or 18 for BPRS) $\times 100$.

To compare standardised total scores and per cent score changes over time between the two treatment groups (i.e. continuing antipsychotics and switching to placebo), meta-regression analyses were employed with a mixed model that included baseline scores as a covariate ${ }^{17}$ (none for per cent score changes), group, time and interaction between group and time as fixed effects. In order to allow for a flexible model rather than specific functional forms such as linear and quadratic, a fixed effect of time in weeks was specified as a categorical predictor. The model also included a random time slope and a random intercept, with covariance matrix structure between random effects specified as unstructured. The number of participants at baseline was used as regression weights in the analysis as we only included studies using an LOCF method. The estimation method for mixed-effect models was restricted maximum likelihood. This method is in accordance with a previous meta-analysis examining symptom trajectories during antipsychotic treatment in treatment-resistant schizophrenia. ${ }^{10}$
As sensitivity analyses, the following studies or arms were excluded from the analyses: studies using BPRS; LAI treatment arms; FGA treatment arms, low-dose antipsychotic treatment arms defined as $\leqslant 80 \%$ of daily defined dose (DDD; available at http://www.whocc.no/atc_ddd_index); placebo treatment arms where participants had been treated with LAI antipsychotics before randomisation; or, studies with a high baseline PANSS or BPRS score because of the criteria for symptom stabilisation (see Table 1 and online Table DS1). A two-tailed $P$-value of $<0.05$ was considered statistically significant for all tests. All statistical analyses were conducted using the IBM SPSS Statistics version 21 .

\section{Results}

\section{Included studies}

A total of 11 studies ${ }^{18-28}$ ( $n=7$ from the studies included in Leucht et al's meta-analysis; $n=4$ from the additional literature search) involving 2826 participants $(n=1618$ for antipsychotic treatment; $n=1208$ for placebo treatment) that met eligibility criteria were identified and included in the meta-analysis (Fig. 1). The characteristics of these studies are summarised in Table 1, and stabilisation criteria for each are shown in online Table DS1. All studies were parallel-group RCTs conducted in a double-masked fashion with study duration ranging from 24 to 64 weeks. All patients included in the studies had schizophrenia or schizoaffective disorder and the majority of them had a chronic course of illness. All except one study examined SGAs; three studies included an LAI arm and two a low-dose antipsychotics arm; two studies used the BPRS, neither of which reported which item version of the BPRS was used; three studies included participants treated with LAI antipsychotics before randomisation; and, in three the studies' baseline standardised total score was $>2$. The results of risk of bias assessment are displayed in online Fig. DS1. Also, the sample sizes at each time point in the antipsychotic and placebo treatment groups are shown in online Tables DS2 and DS3, respectively.

\section{Symptom trajectories in antipsychotic versus placebo treatment}

The symptom trajectories of standardised total scores and per cent score changes in the antipsychotic and placebo treatment arms are depicted in Figs 2 and 3, respectively. As indicated in Fig. 2, symptom severity remained nearly unchanged in patients continuing antipsychotic treatment, whereas symptom severity gradually and continuously worsened over time in those switching to placebo treatment; the interaction between group and time was statistically significant $(F=10.5, P<0.0001)$. Similarly, a placebo replacement was associated with a $49.2 \%$ worsening of symptoms from baseline to 52 weeks, in contrast to $10.1 \%$ in those who continued antipsychotic treatment; the interaction was significant $(F=8.34, P<0.0001)$ (Fig. 3).

Sensitivity analyses revealed that the interactions between group and time remained statistically significant after excluding studies with use of BPRS $(n=2)$, LAI arms $(n=3)$, FGA arms $(n=2)$, low-dose antipsychotic arms $(n=2)$, placebo arms where participants were treated with LAI antipsychotics before randomisation $(n=3)$ or studies with a severe baseline symptom level $(n=3)$ (all $P s<0.0001)$.

\section{Discussion}

\section{Main findings and comparison with findings from other studies}

In patients with stable schizophrenia, we found that continuing antipsychotics significantly reduced symptom exacerbation over 


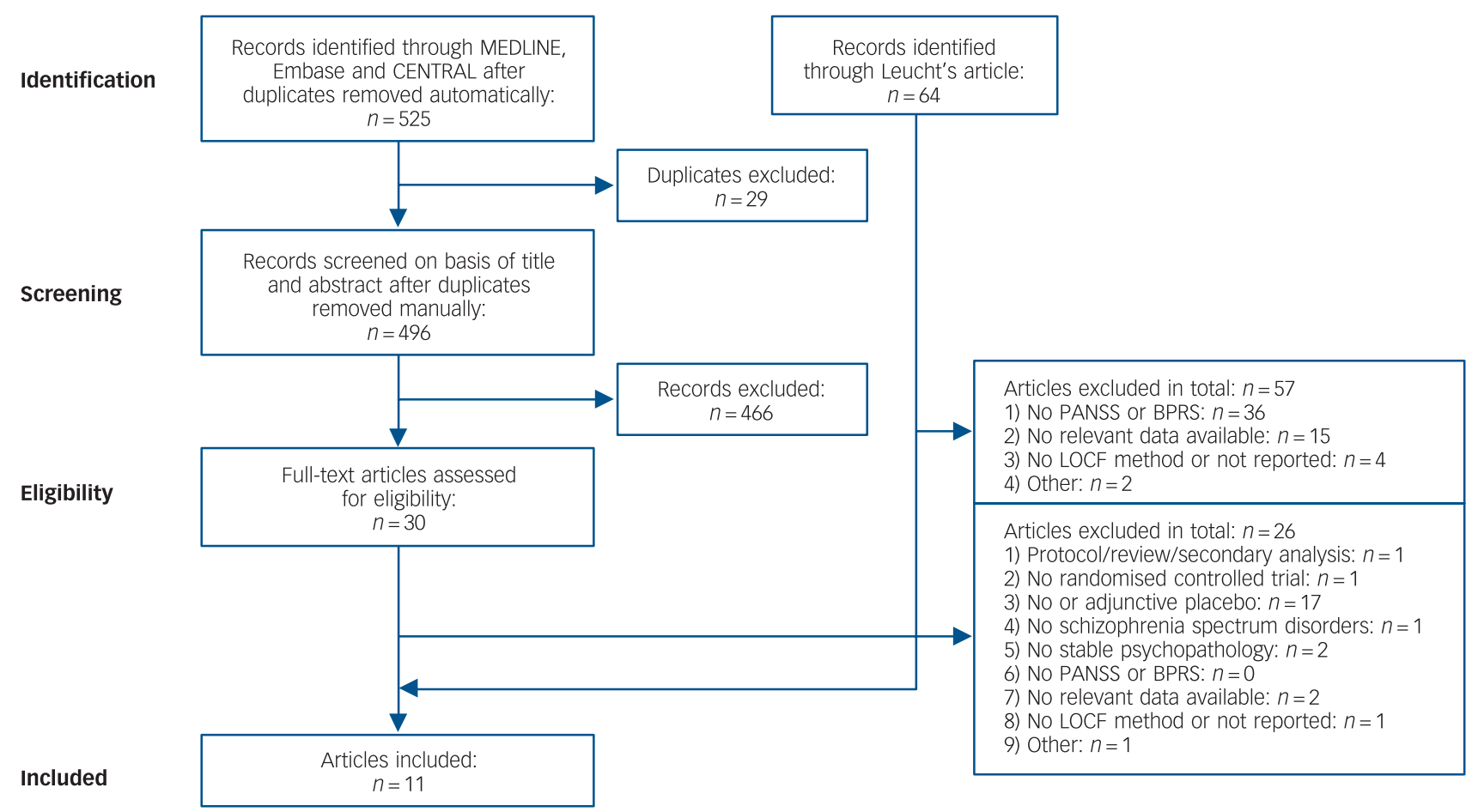

Fig. 1 PRISMA flow diagram of the literature search.

the following year when compared with individuals switched to placebo. Our findings corroborate the evidence from Leucht et al's meta-analysis showing that relapse risk in stable schizophrenia is reduced with antipsychotic maintenance treatment. ${ }^{2}$ However, and most importantly, the current meta-analysis did not employ categorical outcomes that require a specific definition, such as relapse, exacerbation, remission and response, definitions that frequently vary between studies. Instead, we used total scores from the two most commonly employed rating scales in clinical trials involving schizophrenia. This allowed us to treat the data as a continuous variable and compare the trajectory of symptom changes between the two treatment groups with meta-regression analyses. Moreover, all included RCTs were conducted in a double-masked fashion and used placebo treatment rather than no treatment, even though these were not part of the eligibility criteria for the studies. This minimises the chance of performance biases, such as participant and assessor expectations, which can significantly influence results.

Our findings shed light on the importance of using symptom severity rather than relapse to evaluate long-term outcomes in schizophrenia; in contrast to Leucht et al's meta-analysis where the relapse rate between 7 and 12 months was as high as $27 \%$ even in patients continuing antipsychotic treatment, ${ }^{2}$ our meta-analysis revealed no essential increment in symptom severity in those continuing antipsychotic treatment over 1 year. Further, the current meta-analysis demonstrated that the risk commences almost immediately and progresses in a linear fashion following antipsychotic discontinuation. From a clinical perspective, it argues for immediate and continuous monitoring; within the year following discontinuation there is no time point at which symptom exacerbation reaches a plateau. This said, it should be stressed that not all patient trajectories are the same. The mean score of overall symptom severity in patients receiving placebo can be affected by the scores in those who relapsed; in fact, a significant association between relapse rates and standardised symptom score changes was observed in patients undergoing placebo treatment (online Fig. DS2).

\section{Considerations when interpreting our findings}

It is worth highlighting once again that the majority of studies included in the current meta-analysis involved patients whose conditions were more chronic in nature; accordingly, the findings cannot be extrapolated to other populations such as those with first-episode schizophrenia. However, Leucht et al have indicated a greater relapse risk with placebo versus ongoing antipsychotic treatment in a subgroup of patients with first-episode schizophrenia. ${ }^{2}$ In addition, Gitlin et al have demonstrated that the rate of symptom exacerbation continues to increase over 2 years in individuals with remitted recent-onset schizophrenia who discontinue treatment, ${ }^{29}$ which is also supported in a recent study and systematic review. ${ }^{30,31}$ Nonetheless, this does not rule out the following possibilities: (a) a specific group of patients may not require antipsychotic maintenance treatment following resolution of an acute psychotic episode; (b) antipsychotics can be reduced rather than totally discontinued. Naturalistic studies with a follow-up duration of 10 years or more have indicated that approximately one-third of patients with schizophrenia can remain stable in the absence of antipsychotic treatment. ${ }^{32-34}$ To date, though, no clinical factors have been definitively established that predict those individuals who will not experience relapse following antipsychotic discontinuation. Regarding the second issue, there is still ongoing debate as to whether antipsychotic dose reduction is feasible in terms of relapse prevention; ${ }^{1}$ however, some studies have reported benefits with antipsychotic dose reduction, including improvement in motor and cognitive sideeffects. ${ }^{35,36}$ Clinicians should continue to make efforts to minimise side-effects during the long-term antipsychotic treatment of schizophrenia.

Given that the vast majority of included studies examined SGAs and a meta-analysis has shown that SGAs are superior to FGAs in terms of relapse prevention, ${ }^{4}$ it remains unknown if FGAs demonstrate the same magnitude of separation over time versus placebo as SGAs. Another important issue is the type of antipsychotic formulation participants received prior to randomisation. 


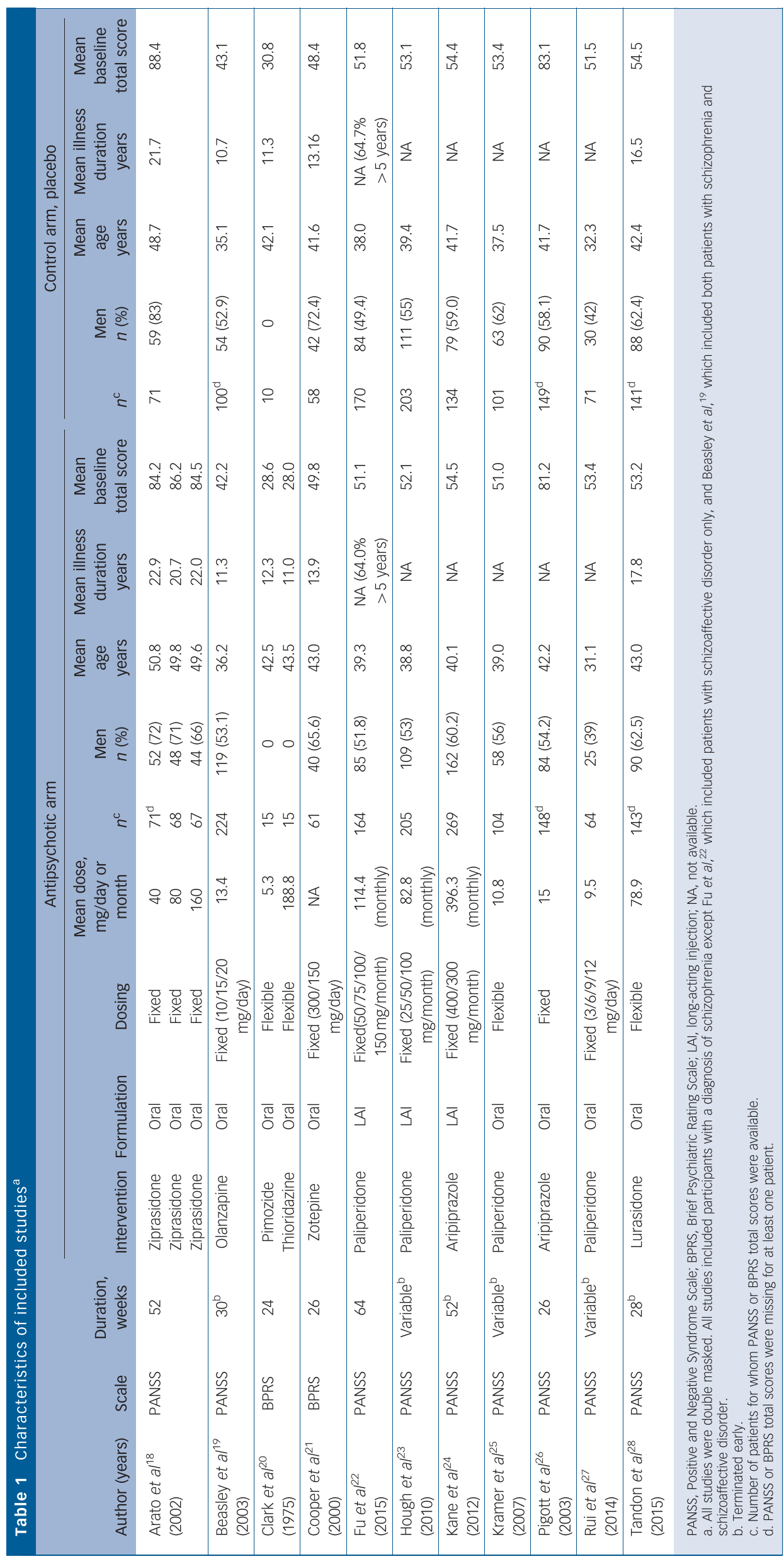




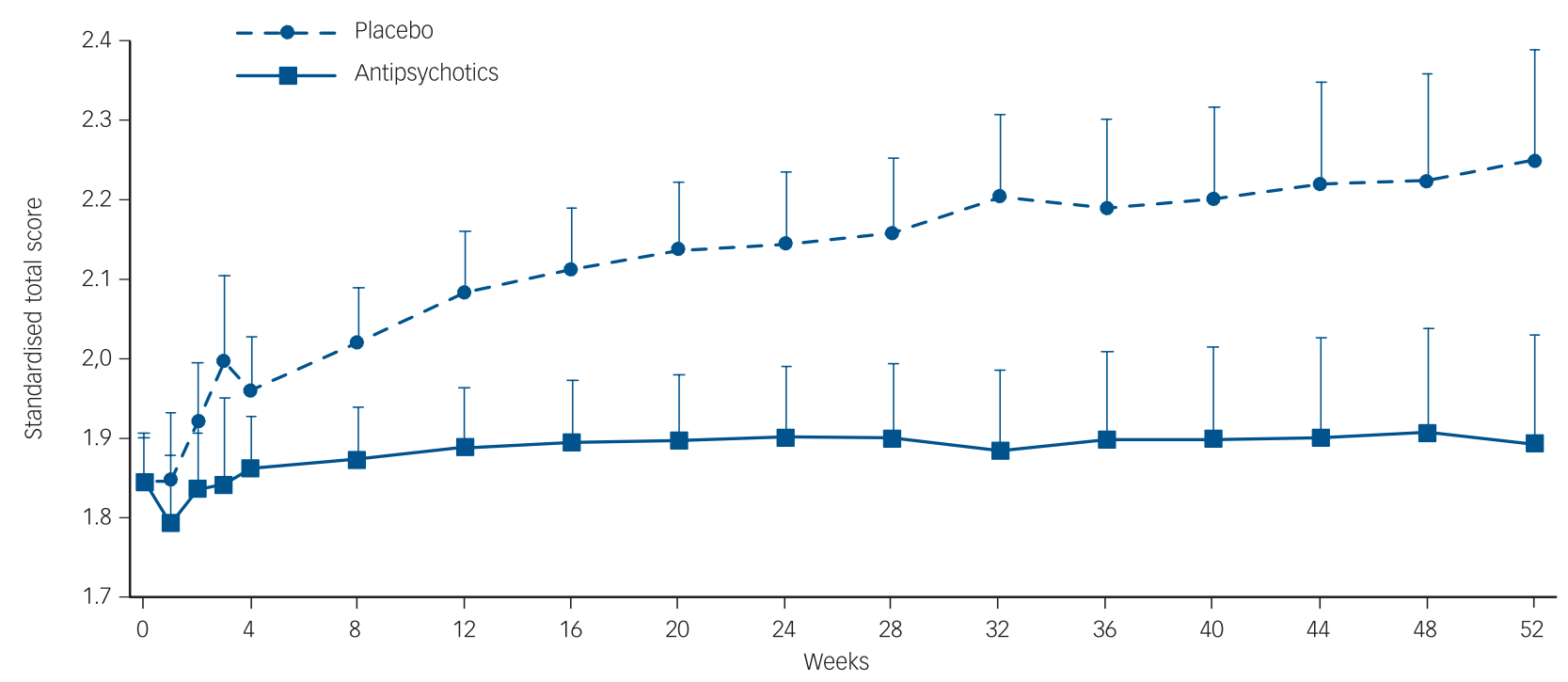

Fig. 2 Symptom trajectories indicated by standardised total score in patients with stable schizophrenia continuing antipsychotics $(n=1618)$ versus switching to placebo $(n=1208)$.

The mixed-model analysis revealed a significant interaction between group and time $(F=10.5, P<0.0001)$. The $y$-axis indicates least squares means of standardised total scores. Error bars denote $95 \%$ confidence intervals.

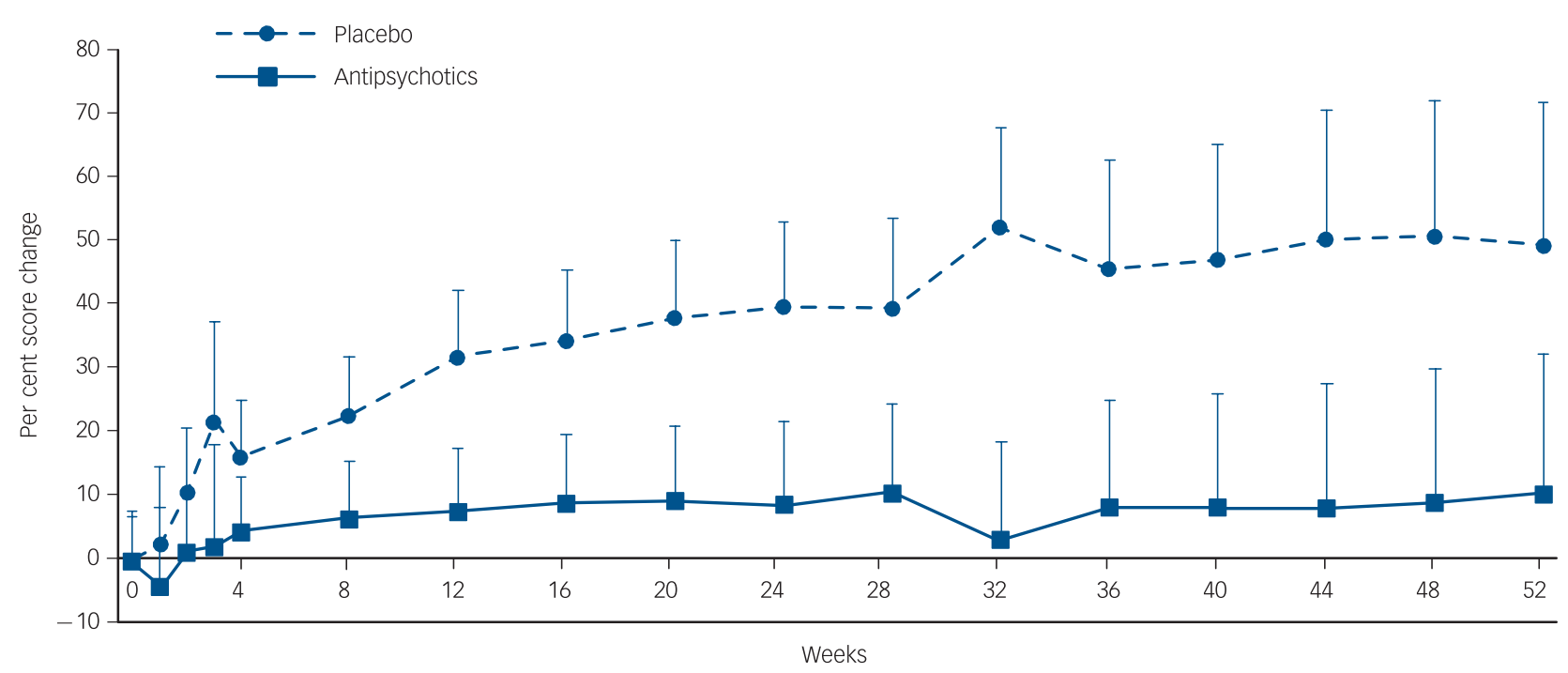

Fig. 3 Symptom trajectories indicated by per cent score change in patients with stable schizophrenia continuing antipsychotics $(n=1618)$ versus switching to placebo $(n=1208)$.

The mixed-model analysis revealed a significant interaction between group and time $(F=8.34, P<0.0001)$. The $y$-axis indicates least squares means of per cent score changes. Error bars denote $95 \%$ confidence intervals.

Hypothetically, for patients treated with LAIs antipsychotic activity will persist for a longer interval owing to their prolonged plasma half-lives, which may alter symptom trajectories in the placebo treatment group even if participants received an abrupt switch to placebo. A previous meta-analysis showed that abrupt withdrawal of antipsychotics was related to a higher relapse rate compared with gradual discontinuation, ${ }^{37}$ although Leucht et al's metaanalysis demonstrated no significant difference in relapse reduction between immediate and gradual antipsychotic discontinuation. ${ }^{2}$ Indeed, all studies except one included in the current meta-analysis appeared to switch to placebo immediately; however, when limiting the analyses to studies where gradual antipsychotic discontinuation was adopted or participants had been treated with LAI antipsychotics before randomisation $(n=4)$, the findings remained unchanged $(P s>0.0001)$, suggesting that symptom severity may increase even if antipsychotic treatment is discontinued gradually.

\section{Limitations}

As expected, there was considerable variation between the studies included regarding criteria for both symptom and treatment stabilisation (see online Table DS1). This was reflected, for example, in differences in baseline symptom severity among studies; the majority of the studies had a PANSS total score in the range of 55, representing 'borderline mentally ill,, 38 although three studies had relatively severe symptomatology (Table 1). These three studies also included less stringent criteria for 
stabilisation and did not include a stabilisation phase where patients were treated with an antipsychotic for a defined period to confirm symptom and/or treatment stabilisation before treatment randomisation (online Table DS1). This was reflected in higher baseline scores but as part of our data analysis we controlled for baseline symptom severity and excluded the three studies for sensitivity analysis; however, results remained the same.

Since only one study had a follow-up period of longer than 1 year, we could not examine the time course of symptoms beyond 52 weeks. This underscores the paucity of longer-term RCTs comparing clinical outcomes between antipsychotics and placebo, although there are ethical concerns related to prolonged placebo exposure. Among the 11 studies included, three adopted a variable duration design and six were terminated early as they involved trials of new antipsychotics where efficacy was established in the course of interim analyses. Long-term antipsychotic exposure may be associated with unfavourable consequences, including structural brain changes and dopamine receptor supersensitivity (see the recent argument on long-term prophylactic use of antipsychotics ${ }^{39}$ ). Moreover, Wunderink et al have reported that patients with remitted first-episode psychosis (approximately $70 \%$ with a diagnosis of schizophrenia spectrum disorders) receiving antipsychotic reduction/discontinuation are more likely to achieve functional remission over 7 years compared with those continuing antipsychotic treatment ${ }^{40}$ despite shorter-term clinical outcomes being worse. ${ }^{41}$ Thus, our findings cannot speak to what might occur beyond 1 year, nor do they address other measures such as functional outcome. Given that functional outcome is important in discussions of recovery in patients with schizophrenia, ${ }^{42}$ clinicians must balance symptomatic and functional outcomes.

Other limitations need to be noted as well. First, the number of included studies is relatively small compared with the metaanalysis of Leucht et al ( $n=11$ v. 65); whereas that meta-analysis involved approximately 6500 participants, ours included 2826. Second, six studies reported only baseline and end-point data, which resulted in fluctuations in sample sizes across time (online Tables DS2 and DS3). Third, we only included studies using a LOCF method because of our intention to add sample weights in the meta-regression analyses. An additional reason behind this strategy was the fact that most studies did not report sample size at each time point for PANSS or BPRS total scores. Thus, we excluded five studies that used either an observed case analysis $(n=1)$ or did not report which approach they adopted $(n=4)$ (Fig. 1). This inclusion criterion may limit the generalisability of our findings, although a previous meta-analysis examining the time course of symptom improvement in antipsychotic treatment demonstrated no difference in rates of score changes over time between the two methods. ${ }^{8}$ Fourth, among five studies reporting PANSS subscale scores (four studies had solely baseline and end-point data, and one study had a third time point), only two studies shared the same time points other than baseline, which prevented us from examining trajectories of different symptom domains such as positive and negative symptoms. Finally, our findings cannot be applied to clozapine, the sole antipsychotic medication approved for treatment-resistant schizophrenia, based on evidence that its time course of symptom improvement differs from other antipsychotics. ${ }^{10,11}$

\section{Implications}

In conclusion, the present meta-analysis indicates that patients with stable schizophrenia who continue antipsychotic treatment demonstrate ongoing symptom stabilisation over a 1-year interval, in contrast to a trajectory of continuous symptom exacerbation observed in patients receiving placebo. This highlights the clinical benefits of antipsychotic maintenance treatment, at least in chronic schizophrenia, as well as the necessity of immediate and ongoing monitoring for clinical worsening in the face of antipsychotic discontinuation. However, given that the current meta-analysis does not address symptomatic outcome beyond 1 year, or functional outcome, clinicians should make decisions on the long-term antipsychotic treatment of schizophrenia based on individual patient needs. It has been suggested that there is a subgroup of individuals who do not require ongoing antipsychotic treatment, ${ }^{32,43}$ but who these individuals are or when it is safe to discontinue maintenance treatment in those that remain stabilised, represent unanswered questions.

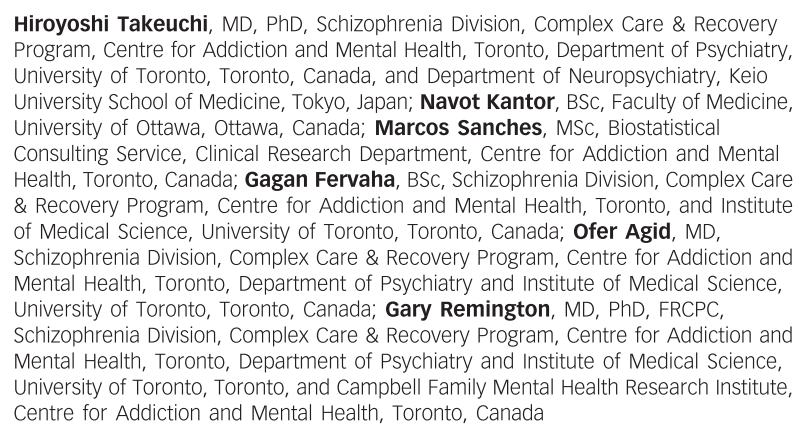

Correspondence: Hiroyoshi Takeuchi, Schizophrenia Division, Complex Care \& Recovery Program, Centre for Addiction and Mental Health, 250 College Street, Toronto, Ontario, M5T 1R8, Canada. Email: hirotak@dk9.so-net.ne.jp

First received 6 Apr 2016, final revision 5 Dec 2016, accepted 6 Mar 2017

\section{Funding}

H.T. is supported through the Canadian Institutes of Health Research (CIHR) fellowship programme. This funding source had no role in study design, statistical analysis or interpretation of findings, or in manuscript preparation or submission for publication. H.T. has received fellowship grants from the Centre for Addiction and Mental Health (CAMH) Foundation, the Japanese Society of Clinical Neuropsychopharmacology and the Astellas Foundation for Research on Metabolic Disorders.

\section{Acknowledgements}

The authors thank Ms Sadhana Thiyanavadivel for her kind help.

\section{References}

1 Takeuchi $H$, Suzuki T, Uchida $H$, Watanabe $K$, Mimura M. Antipsychotic treatment for schizophrenia in the maintenance phase: a systematic review of the guidelines and algorithms. Schizophr Res 2012; 134: 219-25.

2 Leucht S, Tardy M, Komossa K, Heres S, Kissling W, Salanti G, et al. Antipsychotic drugs versus placebo for relapse prevention in schizophrenia: a systematic review and meta-analysis. Lancet 2012; 379: 2063-71.

3 Gleeson JFM, Alvarez-Jimenez M, Cotton SM, Parker AG, Hetrick S. A systematic review of relapse measurement in randomized controlled trials of relapse prevention in first-episode psychosis. Schizophr Res 2010; 119 : 79-88.

4 Kishimoto T, Agarwal V, Kishi T, Leucht S, Kane JM, Correll CU. Relapse prevention in schizophrenia: a systematic review and meta-analysis of second-generation antipsychotics versus first-generation antipsychotics. Mol Psychiatry 2013; 18: 53-66.

5 Kishimoto T, Robenzadeh A, Leucht C, Leucht S, Watanabe K, Mimura M, et al. Long-acting injectable vs oral antipsychotics for relapse prevention in schizophrenia: a meta-analysis of randomized trials. Schizophr Bull 2014; 40: 192-213.

6 Kay SR, Fiszbein A, Opler LA. The positive and negative syndrome scale (PANSS) for schizophrenia. Schizophr Bull 1987; 13: 261-76.

7 Overall JE, Gorham DR. The Brief Psychiatric Rating Scale. Psychol Rep 1962; 10: $799-812$. 
8 Agid O, Kapur S, Arenovich T, Zipursky RB. Delayed-onset hypothesis of antipsychotic action: a hypothesis tested and rejected. Arch Gen Psychiatry 2003; 60: 1228-35.

9 Sherwood $M$, Thornton $A E$, Honer WG. A meta-analysis of profile and time-course of symptom change in acute schizophrenia treated with atypical antipsychotics. Int J Neuropsychopharmacol 2006; 9: 357-66.

10 Suzuki T, Remington G, Arenovich T, Uchida H, Agid O, Graff-Guerrero A, et al. Time course of improvement with antipsychotic medication in treatmentresistant schizophrenia. Br J Psychiatry 2011; 199: 275-80.

11 Sherwood $M$, Thornton $A E$, Honer WG. A quantitative review of the profile and time course of symptom change in schizophrenia treated with clozapine. J Psychopharmacol 2012; 26: 1175-84.

12 Moher D, Liberati A, Tetzlaff J, Altman DG. Preferred reporting items for systematic reviews and meta-analyses: the PRISMA statement. BMJ 2009; 339: b2535

13 Higgins JPT, Green S. Cochrane Handbook for Systematic Reviews of Interventions. The Cochrane Collaboration, 2011 (http://handbook.cochrane.org)

14 Leucht S, Davis JM, Engel RR, Kane JM, Wagenpfeil S. Defining 'response' in antipsychotic drug trials: recommendations for the use of scale-derived cutoffs. Neuropsychopharmacology 2007; 32: 1903-10.

15 Obermeier M, Mayr A, Schennach-Wolff R, Seemüller F, Möller H-J, Riedel M. Should the PANSS be rescaled? Schizophr Bull 2010; 36: 455-60.

16 Leucht S, Kissling W, Davis JM. The PANSS should be rescaled. Schizophr Bull 2010; 36: 461-2.

17 Furukawa TA, Levine SZ, Tanaka S, Goldberg Y, Samara M, Davis JM, et al. Initial severity of schizophrenia and efficacy of antipsychotics: participantlevel meta-analysis of 6 placebo-controlled studies. JAMA Psychiatry 2015; 72: $14-21$.

18 Arato M, O'Connor R, Meltzer HY. A 1-year, double-blind, placebo-controlled trial of ziprasidone 40,80 and $160 \mathrm{mg}$ /day in chronic schizophrenia: the Ziprasidone Extended Use in Schizophrenia (ZEUS) study. Int Clin Psychopharmacol 2002; 17: 207-15

19 Beasley CM, Sutton VK, Hamilton SH, Walker DJ, Dossenbach M, Taylor CC, et al. A double-blind, randomized, placebo-controlled trial of olanzapine in the prevention of psychotic relapse. J Clin Psychopharmacol 2003; 23: 582-94.

20 Clark ML, Huber WK, Hill D, Wood F, Costiloe JP. Pimozide in chronic schizophrenic outpatients. Dis Nerv Syst 1975; 36: 137-41.

21 Cooper SJ, Butler A, Tweed J, Welch C, Raniwalla J. Zotepine in the prevention of recurrence: a randomised, double-blind, placebo-controlled study for chronic schizophrenia. Psychopharmacology (Berl) 2000; 150 237-43.

22 Fu D-J, Turkoz I, Simonson RB, Walling DP, Schooler NR, Lindenmayer J-P, et al. Paliperidone palmitate once-monthly reduces risk of relapse of psychotic, depressive, and manic symptoms and maintains functioning in a double-blind, randomized study of schizoaffective disorder. J Clin Psychiatry 2015; 76: 253-62.

23 Hough D, Gopal S, Vijapurkar U, Lim P, Morozova M, Eerdekens M Paliperidone palmitate maintenance treatment in delaying the time-torelapse in patients with schizophrenia: a randomized, double-blind, placebo-controlled study. Schizophr Res 2010; 116: 107-17.

24 Kane JM, Sanchez R, Perry PP, Jin N, Johnson BR, Forbes RA, et al. Aripiprazole intramuscular depot as maintenance treatment in patients with schizophrenia: a 52-week, multicenter, randomized, double-blind, placebocontrolled study. J Clin Psychiatry 2012; 73: 617-24.

25 Kramer M, Simpson G, Maciulis V, Kushner S, Vijapurkar U, Lim P, et al. Paliperidone extended-release tablets for prevention of symptom recurrence in patients with schizophrenia: a randomized, double-blind, placebocontrolled study. J Clin Psychopharmacol 2007; 27: 6-14.

26 Pigott TA, Carson WH, Saha AR, Torbeyns AF, Stock EG, Ingenito GG. Aripiprazole for the prevention of relapse in stabilized patients with chronic schizophrenia: a placebo-controlled 26-week study. J Clin Psychiatry 2003; 64: 1048-56.

27 Rui Q, Wang $Y$, Liang S, Liu Y, Wu Y, Wu Q, et al. Relapse prevention study of paliperidone extended-release tablets in Chinese patients with schizophrenia. Prog Neuropsychopharmacol Biol Psychiatry 2014; 53C 45-53.

28 Tandon R, Cucchiaro J, Phillips D, Hernandez D, Mao Y, Pikalov A, et al. A double-blind, placebo-controlled, randomized withdrawal study of lurasidone for the maintenance of efficacy in patients with schizophrenia. J Psychopharmacol 2016; 30: 69-77.

29 Gitlin M, Nuechterlein K, Subotnik KL, Ventura J, Mintz J, Fogelson DL, et al. Clinical outcome following neuroleptic discontinuation in patients with remitted recent-onset schizophrenia. Am J Psychiatry 2001; 158: 1835-42.

30 Zipursky RB, Menezes NM, Streiner DL. Risk of symptom recurrence with medication discontinuation in first-episode psychosis: a systematic review. Schizophr Res 2014; 152: 408-14.

31 Mayoral-van Son J, de la Foz VO-G, Martinez-Garcia O, Moreno T, Parrilla-Escobar $M$, Valdizan EM, et al. Clinical outcome after antipsychotic treatment discontinuation in functionally recovered first-episode nonaffective psychosis individuals. J Clin Psychiatry 2016; 77: 492-500.

32 Harrow M, Jobe TH, Faull RN. Do all schizophrenia patients need antipsychotic treatment continuously throughout their lifetime? A 20-year longitudinal study. Psychol Med 2012; 42: 2145-55.

33 Moilanen J, Haapea M, Miettunen J, Jääskeläinen E, Veijola J, Isohanni M, et al. Characteristics of subjects with schizophrenia spectrum disorder with and without antipsychotic medication - a 10-year follow-up of the Northern Finland 1966 Birth Cohort study. Eur Psychiatry 2013; 28: 53-8.

34 Wils RS, Gotfredsen DR, Hjorthøj C, Austin SF, Albert N, Secher RG, et al. Antipsychotic medication and remission of psychotic symptoms 10 years after a first-episode psychosis. Schizophr Res 2017; 182: 42-8.

35 Inderbitzin LB, Lewine RR, Scheller-Gilkey G, Swofford CD, Egan GJ, Gloersen $\mathrm{BA}$, et al. A double-blind dose-reduction trial of fluphenazine decanoate for chronic, unstable schizophrenic patients. Am J Psychiatry 1994; 151: 1753-9.

36 Takeuchi H, Suzuki T, Remington G, Bies RR, Abe T, Graff-Guerrero A, et al. Effects of risperidone and olanzapine dose reduction on cognitive function in stable patients with schizophrenia: an open-label, randomized, controlled, pilot study. Schizophr Bull 2013; 39: 993-8.

37 Viguera AC, Baldessarini RJ, Hegarty JD, van Kammen DP, Tohen M. Clinical risk following abrupt and gradual withdrawal of maintenance neuroleptic treatment. Arch Gen Psychiatry 1997; 54: 49-55.

38 Leucht S, Kane JM, Kissling W, Hamann J, Etschel E, Engel RR. What does the PANSS mean? Schizophr Res 2005; 79: 231-8.

39 Murray RM, Quattrone D, Natesan S, van Os J, Nordentoft M, Howes O, et al. Should psychiatrists be more cautious about the long-term prophylactic use of antipsychotics? Br J Psychiatry 2016; 209: 361-5.

40 Wunderink L, Nieboer RM, Wiersma D, Sytema S, Nienhuis FJ. Recovery in remitted first-episode psychosis at 7 years of follow-up of an early dose reduction/discontinuation or maintenance treatment strategy: long-term follow-up of a 2-year randomized clinical trial. JAMA Psychiatry 2013; 70: 913-20.

41 Wunderink L, Nienhuis FJ, Sytema S, Slooff CJ, Knegtering R, Wiersma D. Guided discontinuation versus maintenance treatment in remitted firstepisode psychosis: relapse rates and functional outcome. J Clin Psychiatry 2007; 68: 654-61.

42 Jaaskelainen E, Juola P, Hirvonen N, McGrath JJ, Saha S, Isohanni M, et al. A systematic review and meta-analysis of recovery in schizophrenia. Schizophr Bull 2013; 39: 1296-306

43 Harrow M, Jobe TH. Does long-term treatment of schizophrenia with antipsychotic medications facilitate recovery? Schizophr Bull 2013; 39 : 962-5. 